\title{
Initial nucleon structure results with chiral quarks at the physical point
}

\author{
S. Syritsyn ${ }^{* a}$, T. Blum ${ }^{a, b}$, M. Engelhardt ${ }^{c}$, J. Green ${ }^{d}$, T. Izubuchi ${ }^{a}$, C. Jung ${ }^{a}$, \\ S. Krieg ${ }^{e}$, M. Lin ${ }^{f}$, S. Meinel ${ }^{a, g}$, J. Negele ${ }^{h}$, S. Ohta ${ }^{i, j, a}$, A. Pochinsky ${ }^{h}$, \\ E. Shintani ${ }^{a, k}$ (RBC and LHP collaborations) \\ ${ }^{a}$ RIKEN/BNL Research Center, Brookhaven National Laboratory, Upton, NY, 11973, USA \\ ${ }^{b}$ Physics Department, University of Connecticut, Storrs, CT 06269, USA \\ ${ }^{c}$ Department of Physics, New Mexico State University, Las Cruces, NM 88003, USA \\ ${ }^{d}$ Institut für Kernphysik, Johannes Gutenberg-Universität Mainz, D-55099 Mainz, Germany \\ ${ }^{e}$ Bergische Universität Wuppertal, D-42119 Wuppertal, Germany and \\ IAS, Jülich Supercomputing Centre, Forschungszentrum Jülich, D-52425 Jülich, Germany \\ ${ }^{f}$ Computational Science Center, Brookhaven National Laboratory, Upton, NY 11973, USA \\ ${ }^{g}$ Department of Physics, University of Arizona, Tucson, AZ 85721, USA \\ ${ }^{h}$ Massachusetts Institute of Technology, Cambridge, MA 02139, USA \\ ${ }^{i}$ Institute of Particle and Nuclear Studies, KEK, Tsukuba, Ibaraki 3050801, Japan \\ ${ }^{j}$ Department of Particle and Nuclear Physics, SOKENDAI, Hayama, Kanagawa, 2400193, Japan \\ ${ }^{k}$ PRISMA Cluster of Excellence, Institut für Kernphysik and Helmholtz Institute Mainz, Johannes \\ Gutenberg-Universität Mainz, D-55099 Mainz, Germany \\ E-mail: ssyritsyn@quark.phy.bnl.gov
}

\begin{abstract}
We report initial nucleon structure results computed on lattices with $2+1$ dynamical Möbius domain wall fermions at the physical point generated by the RBC and UKQCD collaborations. At this stage, we evaluate only connected quark contributions. In particular, we discuss the nucleon vector and axial-vector form factors, nucleon axial charge and the isovector quark momentum fraction. From currently available statistics, we estimate the stochastic accuracy of the determination of $g_{A}$ and $\langle x\rangle_{u-d}$ to be around $10 \%$, and we expect to reduce that to $5 \%$ within the next year. To reduce the computational cost of our calculations, we extensively use acceleration techniques such as low-eigenmode deflation and all-mode-averaging (AMA). We present a method for choosing optimal AMA parameters.
\end{abstract}

The 32nd International Symposium on Lattice Field Theory,

23-28 June, 2014

Columbia University New York, NY

\footnotetext{
* Speaker.
} 


\section{Introduction}

Exploring nucleon structure in lattice QCD at the physical point became possible in the recent few years, and many collaborations have started calculations that no longer require chiral extrapolations. This is a major step forward because it eliminates one of the major sources of systematic uncertainty that made difficult validating methods and achieving high precision in lattice QCD.

Nucleon structure calculations at the physical point are extremely demanding. This usually justifies choosing an affordable fermion action such as Wilson [1] or twisted mass [2], even though they do not respect chiral symmetry of QCD. However, a number of nuclear and high energy physics problems, e.g., proton decay and neutron oscillations, require chiral symmetry for computing corresponding nucleon matrix elements, and one has to use substantially more expensive domain wall or overlap fermion actions. Studying nucleon structure, the nucleon vector and axial vector form factors in particular, with these actions is therefore a necessary stepping stone for developing methods to facilitate calculations that will preserve chiral symmetry.

In this report, we show our initial results from computing nucleon structure directly at the physical point using gauge configurations generated with the $N_{f}=2+1$ dynamical domain wall fermion action [3]. We use various improvement techniques, which we discuss in Sec. 2, and present initial, low-statistics results for the nucleon form factors, axial charge and quark momentum fraction in Sec. 3.

\section{Methodology}

For this initial study with chiral quarks at the physical point, we use one ensemble of gauge configurations generated by the RBC/UKQCD collaborations with the inverse lattice spacing $a^{-1}=$ $1.730(4) \mathrm{GeV}$ and the pion mass $m_{\pi}=139.2(4) \mathrm{MeV}$ [3]. This ensemble is generated with the Iwasaki gauge action and the Möbius Domain Wall fermion (MDWF) action for $N_{f}=2+1$ quarks. The Möbius formulation [4] of chiral fermions allows one to shorten the fifth ("flavor") dimension of the traditional domain wall fermion action without increasing the quark residual mass. The lattice size is $48^{3} \times 96$, corresponding to $m_{\pi} L=3.86$, which should be sufficient to substantially suppress finite volume effects, as known from the meson sector.

The main challenge in computing hadron observables with domain wall-like actions is the cost of calculating light quark propagators, especially at the physical point. We use two methods to accelerate our calculations: acceleration of the conjugate gradient algorithm (CG) with loweigenmode deflation and improved stochastic sampling, or All-mode Averaging (AMA) [5]. The idea behind AMA is to compute cheap approximate samples with large statistics and then correct for any potential bias by comparing a subset of approximate samples to exact solutions,

$$
\begin{gathered}
\langle\mathscr{O}\rangle_{\text {imp }}=\left\langle\mathscr{O}_{\text {approx }}\right\rangle_{N_{\text {approx }}}+\langle\Delta \mathscr{O}\rangle_{N_{\text {exact }}, \quad \Delta \mathscr{O}=\mathscr{O}_{\text {exact }}-\mathscr{O}_{\text {approx }}}, \\
\left(\delta \mathscr{O}_{\text {imp }}\right)^{2}=\frac{1}{N_{\text {approx }}} \operatorname{Var}\left\{\mathscr{O}_{\text {approx }}\right\}+\frac{1}{N_{\text {exact }}} \operatorname{Var}\{\Delta \mathscr{O}\}
\end{gathered}
$$

One usually takes a large number of approximate samples at different locations on the same lattice configuration, exploiting the translational invariance of the QCD ensemble average, while for $\Delta \mathscr{O}$ smaller statistics is sufficient to estimate the difference arising from the approximation. We obtain 


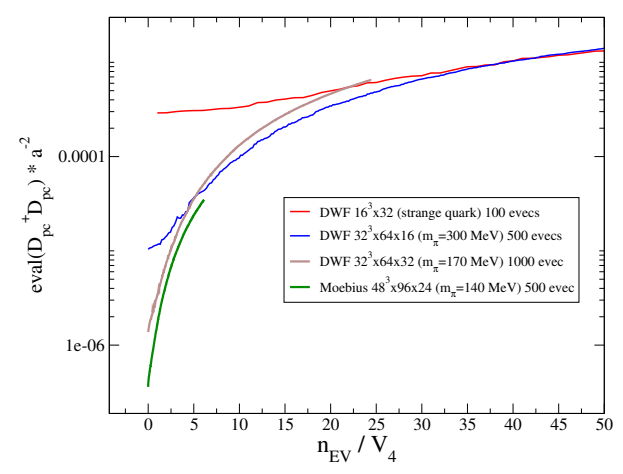

Figure 1: Deflation eigenvalues for different domain wall operators, scaled with volume and lattice cutoff (courtesy of T. Blum, T. Izubuchi, and E. Shintani). The green line corresponds to the current work.
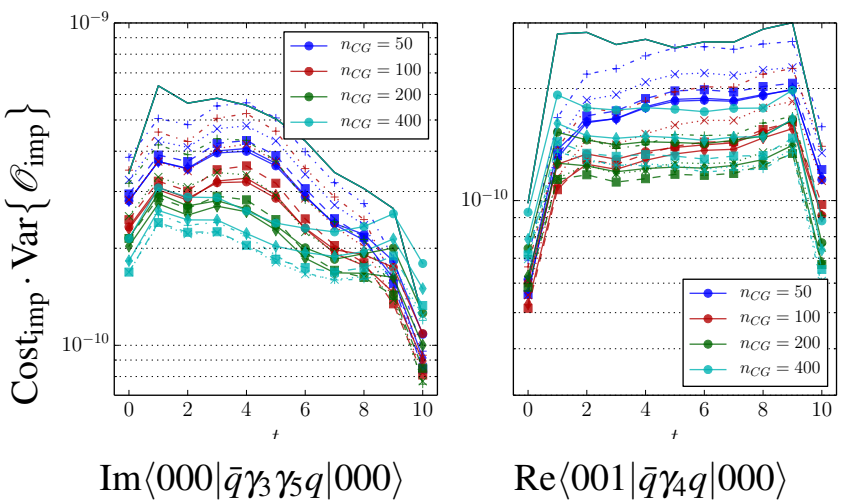

Figure 2: $\quad$ Figure of merit for AMA optimization (see Eq. 2.3) of the nucleon axial charge (left) and charge radius (right) three-point function plateaus. The circles, diamonds, squares, "x" and " +" correspond to $N_{\text {approx }} / N_{\text {exact }}=$ $4,8,16,32,64$, respectively, and the solid line corresponds to exact-only samples (no AMA).

approximate samples using quark propagators computed with truncated CG. Low-eigenmode deflation is applied in computing both exact and approximate samples. In addition to accelerating the CG convergence for the exact samples, deflation helps to reduce the bias of approximate samples and make them cheaper by substantially reducing the number $n_{\mathrm{CG}}$ of $\mathrm{CG}$ iterations required for good approximation.

We compute $N_{E V}=500$ lowest eigenmodes for deflation using ARPACK with $n=200$ Chebyshev polynomial acceleration. With the range of deflated eigenvalues $\lambda_{\max } / \lambda_{\min } \approx 10^{2}$ (see Fig. 1), the convergence rate of $C G$ is accelerated by a factor of $\approx 10$, which was confirmed by direct tests. The number of eigenvalues we can use is limited by the total amount of memory available to a single job. In fact, using $(3 \ldots 4) \times$ more eigenvectors, which would span the spectrum between the light and strange quark masses, is expected to improve the AMA efficiency significantly [6].

In order to apply the AMA effectively, one has to choose the optimal approximation (number of truncated CG steps $n_{\mathrm{CG}}$ ) and ratio of exact and approximate samples $N_{\text {approx }} / N_{\text {exact }}$. Such optimization should take into account the change in the precision of the approximate samples, as well as their total cost. We use the product of their stochastic variance and the cost required for their computation, normalized by the cost of a single exact sample, as the figure of merit:

$$
\operatorname{Cost}_{\text {imp }} \cdot \operatorname{Var}\left\{\mathscr{O}_{\text {imp }}\right\} \sim\left(1+\frac{n_{\mathrm{CG}}^{\text {approx }}}{n_{\mathrm{CG}}^{\text {exact }}} \cdot \frac{N_{\text {approx }}}{N_{\text {exact }}}\right) \cdot\left(\operatorname{Var}\{\Delta \mathscr{O}\}+\frac{N_{\text {exact }}}{N_{\text {approx }}} \operatorname{Var}\left\{\mathscr{O}_{\text {approx }}\right\}\right) .
$$

Estimates of this FOM for varying combinations of $n_{\mathrm{CG}}$ and $N_{\text {approx }} / N_{\text {exact }}$ are shown in Fig. 2 for the nucleon three-point functions yielding the axial charge and the charge radius $^{1}$. From that, we derive optimal $N_{\text {approx }} / N_{\text {exact }}=32$ and $n_{\mathrm{CG}}^{\text {approx }}=400$, which reduce stochastic variance by a factor of $\times(2.5 \ldots 3)$.

Excited states in lattice nucleon matrix elements are a major source of systematic error, and one has to study the dependence of nucleon-operator three-point functions on the separation between

\footnotetext{
${ }^{1}$ More precisely, the matrix element of the vector current with the minimal non-zero momentum transfer.
} 


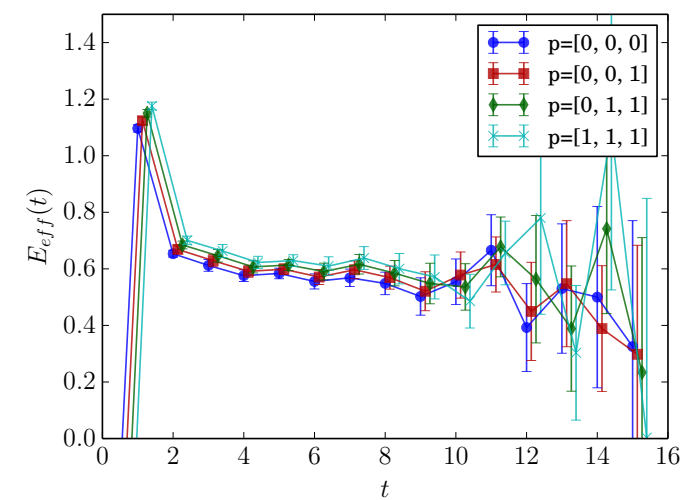

Figure 3: Plateaus for nucleon effective energies for the lowest momenta.

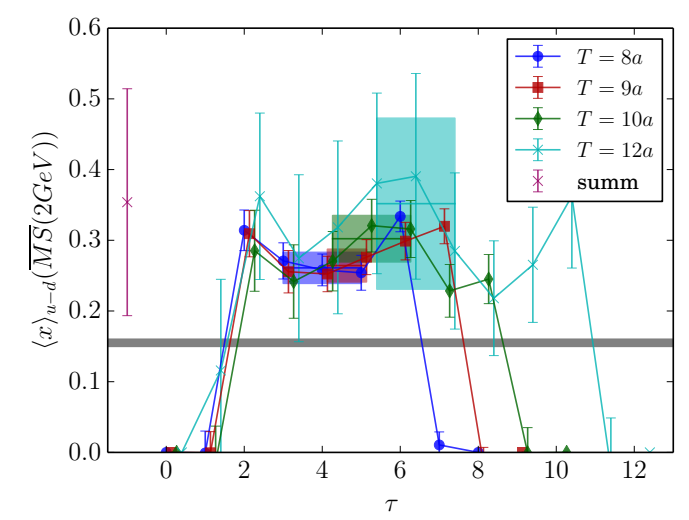

Figure 4: Plateaus of quark momentum fraction $\langle x\rangle_{u-d}$. The renormalization is taken from Ref. [9].

sources and sinks $T=t_{\text {sink }}-t_{\text {source }}$. For this initial study, we select $T / a=8,9,10,12$ corresponding to $0.91,1.03,1.14$, and $1.37 \mathrm{fm}$. Since the statistics are not sufficient for reliable multi-exponential fits, we resort to simpler methods such as summation (e.g., Ref. [7]) to estimate and reduce excitedstate contributions at this stage.

\section{Initial Results}

We have analyzed 20 gauge configurations separated by 80 MD steps to minimize autocorrelations; these configurations are spread evenly over the entire available ensemble except for the first $640 \mathrm{MD}$ steps necessary for thermalization. We compute one exact and 32 approximate samples per configuration. At this initial stage, we focus on nucleon structure "benchmark quantities": the nucleon axial charge, Dirac and Pauli isovector radii, isovector magnetic moment and quark momentum fraction. As shown in Fig. 3, the effective energies $E_{\text {eff }}(t)=\log \left(C_{2 \mathrm{pt}}(t) / C_{2 \mathrm{pt}}(t+1)\right)$ become too noisy after $t=11$, indicating that the source-sink separation $T / a=12$ is the largest one that can be analyzed with the current statistics. In order to extract nucleon form factors from nucleon matrix elements we use standard methods that can be found elsewhere (e.g., see Refs. [8, 9, 1, 10]).

In Figs. 4 and 5 we show quark momentum fraction, vector charge, and axial charge plateaus, their central plateau average values and the values obtained with the summation method [7] (applied to data with all the four separations $T$ ). Although we have not calculated renormalization factors for the momentum fraction for this ensemble, we can use the factors from earlier calculations with Domain Wall fermions with heavier pion masses and the identical lattice spacing [9] to convert bare quantities to the $\overline{\mathrm{MS}}$ scheme at $2 \mathrm{GeV}$. The nucleon isovector quark momentum fraction is known to have substantial contributions from excited states (see, e.g. [1, 11, 12]), and lattice QCD results typically overestimate experiment by 30-60\%. Our initial values for shorter separations also significantly deviate from experiment, while the longest separation $T / a=12$ has insufficient precision to investigate whether it is excited states that cause this systematic effect. The "summation" method yields a value that is consistent with experiment by virtue of its larger statistical uncertainty. 

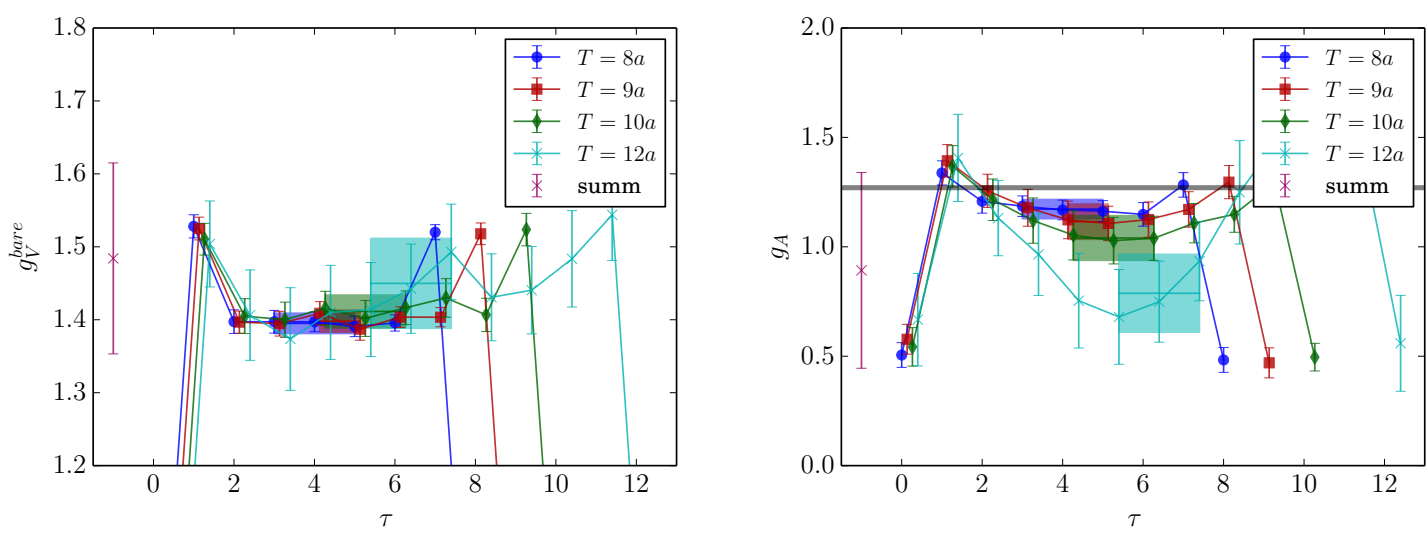

Figure 5: Plateaus of vector (left) and axial (right) charges of the nucleon. The leftmost point represents the "summation" value.

The vector charge plateaus agree for all separations, and we use the central value at $T=$ $8 a$ as an approximate renormalization factor for the vector and axial-vector current operators, $Z_{A}=Z_{V}=\left(\left.g_{V}\right|_{T=8 a}\right)^{-1}$. The value of the axial charge $g_{A}$ is below the experimental value $g_{A}=$ 1.2723(23) [13]. We note that the deviation from the experiment is $(2 \ldots 3) \sigma$, and it can still be attributed to a statistical fluctuation. In addition, the plateaus indicate significant excited state contamination. Although the value from the summation method (Fig. 5(right)) agrees with experiment, the central plateau values move away from it with increasing separation $T$, a behavior that was also observed in other studies close to the physical point [1]. Additional statistics and careful analysis of excited states are required to understand this phenomenon.

The statistics are not yet sufficient to analyze the form factors to extract the radii reliably, especially with larger separations. Since no chiral extrapolation is needed, it is more informative to compare the form factors directly to experiment. In Figure 6 we show the isovector Dirac and Pauli form factors in the region of small momentum transfer $0 \leq Q^{2} \lesssim 0.6 \mathrm{GeV}^{2}$, together with phenomenological fits of experimental data [14]. The Dirac form factor at small separations $T / a=$ $8,9,10$ deviates from the experiment, while the values at the largest separation $T / a=12$, as well as the "summation" value, agree within statistics. This indicates that the deviations are likely caused by excited state contributions, in agreement with earlier findings that the isovector Dirac radius is subject to large excited state effects [1], although more statistics are necessary to make a certain conclusion. The isovector Pauli form factor is in better agreement with phenomenology, although its values are even less precise and the "summation" method yields nearly $100 \%$ uncertainty.

The form factors of the axial-vector current are shown in Fig. 7. The axial form factor $G_{A}\left(Q^{2}\right)$ is interesting for two reasons. First, its forward value $G_{A}(0)=g_{A}$ is the axial charge that has been discussed above; second, its $Q^{2}$-dependence determines the axial radius of the nucleon and plays an important role in the physics of neutrino scattering and meson production. The axial radius, defined similarly to the charge radius, is typically underestimated in lattice calculations by a factor of 2 [15]. From Fig. 7(left) we see that our initial results follow the same pattern: the slope of the axial form factor is substantially smaller than experimental fits. It is not clear yet whether increasing statistics with larger source-sink separations $T / a \geq 10$ will indicate excited state contamination and suggest 

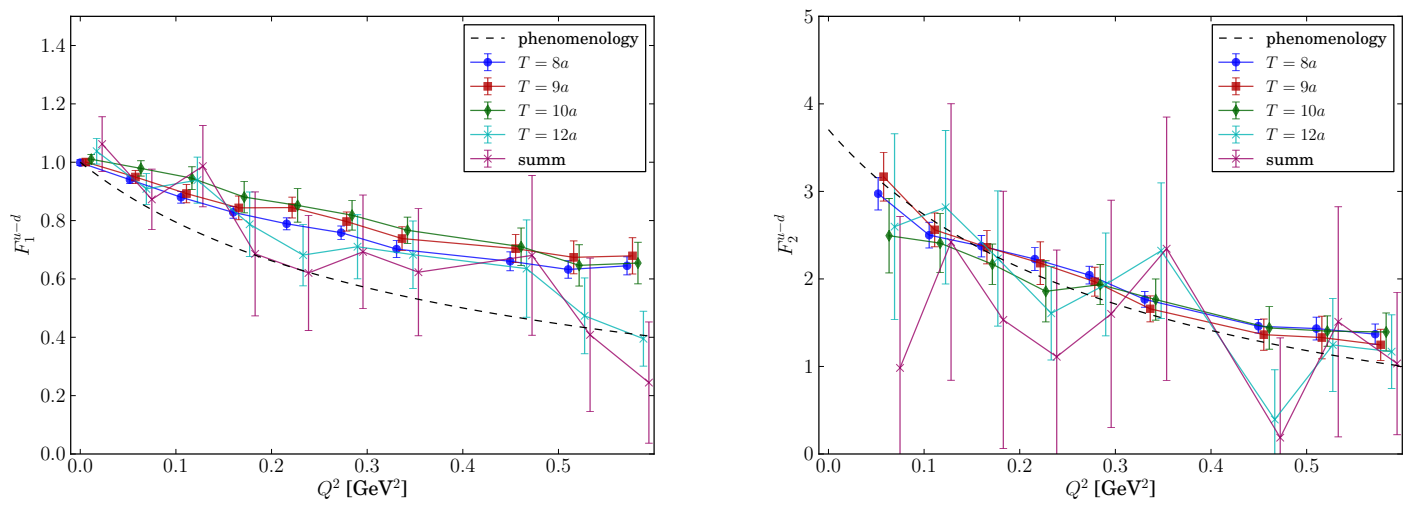

Figure 6: Vector form factors of the nucleon: isovector Dirac (left) and Pauli (right).
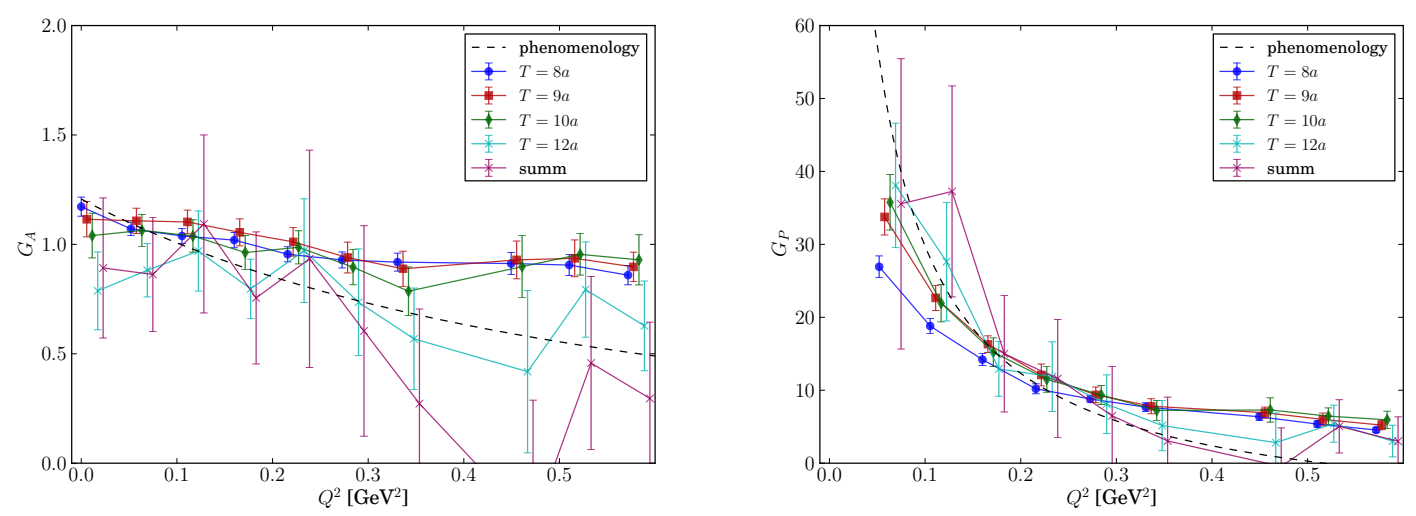

Figure 7: Axial vector form factors of the nucleon: axial (left) and induced pseudoscalar (right).

a solution to this problem; however, comparing $T / a=8,9$ and 10 shows very little dependence on the source-sink separation $T / a$.

The induced pseudoscalar form factor $G_{P}\left(Q^{2}\right)$ (Fig. 7(right)) is relevant for low-energy QCD dynamics. Its value is measured in meson production off nucleons and nuclei, as well as in muon capture experiments [16]. Its low-momentum behavior is believed to be governed by the pion pole $\sim\left(Q^{2}+m_{\pi}^{2}\right)^{-1}$ and therefore is very sensitive to the pion mass. Calculations with the physical pion mass are especially important for this form factor. Our initial results indicate that there are substantial contributions from excited states, and as the source-sink separation increases, we observe better agreement with the phenomenology.

\section{Discussion}

Stochastic uncertainty of the initial results that we report indicates that substantially more statistics will be needed to compute lattice QCD "benchmark" quantities with required precision. Currently, we plan to quadruple statistics. Since, for example, our present stochastic uncertainties for the axial charge and the quark momentum fraction with separation $T=1.14 \mathrm{fm}$ 
are $\left(\delta g_{A}\right)_{T / a=10} \approx 10 \%$ and $\left(\delta\langle x\rangle_{u-d}\right)_{T / a=10} \approx 11 \%$, this will reduce their uncertainties down to $\approx 5 \%$ and make reliable analysis of excited states possible.

Although computing resources increase steadily, and the calculation is fully feasible with the current methodology, we are actively studying approaches to both reduce the cost of computing approximate samples and to make them more precise. An obvious improvement is to simply use more eigenvectors in the CG deflation; however, this approach is limited by the size of the 5D eigenvectors $(\approx 5.6 \mathrm{TiB}$ per configuration). Other possible directions are using approximations to the Möbius fermion operator with shorter $L_{5}$, multigrid methods, and hierarchical deflation [17].

A combination of the improved computing methods and increasing computing resources will allow us to accomplish a reliable calculation of nucleon structure with chirally symmetric action in the near future.

\section{Acknowledgements}

We thank the RBC and UKQCD collaborations for providing us with the gauge configurations. Computations for this work were carried out on facilities of the USQCD Collaboration, which are funded by the Office of Science of the U.S. Department of Energy. This work was supported by RIKEN Foreign Postdoctoral Researcher Program (S.N.S.) and the U.S. Department of Energy (DOE) and the Office of Nuclear Physics under grants DE-FG02-96ER40965 (M.E.) and DE-SC0011090 (J.N.).

\section{References}

[1] J. Green et al., Phys.Lett. B734, 290 (2014).

[2] C. Alexandrou et al., PoS LATTICE2013, 292 (2013).

[3] T. Blum et al., arXiv:1411. 7017 (2014).

[4] R. C. Brower, H. Neff, and K. Orginos, Nucl.Phys.Proc.Suppl. 140, 686 (2005).

[5] T. Blum, T. Izubuchi, and E. Shintani, Phys.Rev. D88, 094503 (2013).

[6] T. Blum and T. Izubuchi, private communications .

[7] N. Mathur, S. Dong, K. Liu, L. Mankiewicz, and N. Mukhopadhyay, Phys.Rev. D62, 114504 (2000).

[8] T. Yamazaki et al., Phys. Rev. D79, 114505 (2009).

[9] Y. Aoki et al., Phys.Rev. D82, 014501 (2010).

[10] J. Green et al., Phys.Rev. D90, 074507 (2014).

[11] G. S. Bali et al., 1408.6850 (2014).

[12] B. Jäger et al., 1311.5804 (2013).

[13] K. Olive et al., Chin.Phys. C38, 090001 (2014).

[14] J. J. Kelly, Phys. Rev. C70, 068202 (2004).

[15] S. Syritsyn, AIP Conf.Proc. 1374, 305 (2011).

[16] S. M. Clayton, AIP Conf.Proc. 1222, 407 (2010).

[17] P. A. Boyle, 1402.2585 (2014). 